\title{
Optimisation des paramètres de dépôt de DLC par ablation laser U.V. de carbone vitreux
}

\author{
J.C. Orlianges, C. Champeaux, A. Catherinot et P. Blondy ${ }^{1}$ \\ Sciences des Procédés Céramiques et de Traitements de Surface, SPCTS, \\ UMR 6638 du CNRS, Faculté des Sciences et Techniques, 123 avenue Albert Thomas, \\ 87060 Limoges cedex, France \\ ${ }^{1}$ Institut de Recherches sur les Communications Optiques et Microondes, IRCOM, \\ UMR 6615 du CNRS, Faculté des Sciences et Techniques, 123 avenue Albert Thomas, \\ 87060 Limoges cedex, France
}

\begin{abstract}
Résumé. Les couches de carbone tétraédrique réalisées par ablation laser (PLD) à forte fluence présentent des propriétés physiques particulièrement intéressantes. Toutefois, dans ces conditions, l'ablation de cibles de graphite s'accompagne de l'éjection de particules solides qui nuisent aux qualités des films. L'utilisation de cibles de carbone vitreux, dans des conditions particulières, permet d'éviter cet écueil. Nous présentons une étude du panache d'ablation par laser $\mathrm{KrF}$ d'une cible de carbone vitreux par imagerie résolue en temps et spectralement, les résultats sont comparés à ceux obtenus avec une cible de graphite.
\end{abstract}

\section{INTRODUCTION}

La dénomination " carbone tétraédrique amorphe " (ta-C) ou encore Diamond-like carbone (DLC) correspond aux couches minces de carbone caractérisées par une forte dureté, une résistivité électrique élevée, une inertie chimique, une bonne transparence dans l'infrarouge et ne présentant aucun ordre structural à grande distance. Les propriétés précédemment citées sont liées, au niveau atomique, à la présence de liaisons chimiques fortes entre les atomes de carbone.

Dans le cas des dépôts réalisés par ablation laser sous vide, l'analyse par microscopie électronique à transmission révèle que la structure des couches de carbone est du type tétraédrique amorphe se composant de grains nanométriques de graphite (carbone $\mathrm{sp}^{2}$ ) dans une matrice de carbone hybridé $\mathrm{sp}^{3}$.

L'optimisation du procédé de dépôt consiste à favoriser la proportion de la phase $\mathrm{sp}^{3}$ (celle du diamant) sur la phase $\mathrm{sp}^{2}$. Par analogie avec ce qui a été démontré dans le cas de dépôts par ares cathodiques filtrés (FCVA), il semble que cette proportion soit limitée à environ $90 \%$ [1].

La réalisation de couche de carbone adamantin (en l'absence d'hydrogène) est soumise à deux conditions:

- le dépôt est réalisé à faible température (entre 25 et $100^{\circ} \mathrm{C}$ ) [2]

- le processus de croissance fait intervenir des atomes ou/et des ions carbone d'énergie élevée.

Ainsi, quand l'énergie des particules éjectées est suffisamment importante pour initier la formation des liaisons de type $\mathrm{sp}^{3}$ et que la mobilité en surface du substrat est réduite du fait de la faible température, les couches présentent les propriétés attendues.

L'incidence de la fluence laser sur les caractéristiques du panache lors de l'ablation d'une cible de graphite par laser à excimères $\mathrm{KrF}(248 \mathrm{~nm})$ et notamment l'évolution de la vitesse et de la concentration d'ions dans le plasma ont été déjà largement étudiées $[3,4]$. Ces résultats, corrélés à des analyses détaillées (spectroscopie Raman, XPS, EELS) des films déposés, permettent d'en déduire que la proportion de liaisons $\mathrm{sp}^{3}$ dans les films dépend fortement de l'énergie cinétique des ions dans le panache et donc de la fluence laser au niveau de la cible. Ceci est corroboré par les résultats obtenus sur des dépôts réalisés par faisceaux d'ions sélectionnés en masse ou par arc filtré qui ont permis d'étudier l'évolution de la fraction de carbone $\mathrm{sp}^{3}$ en fonction de l'énergie des ions $[5,6,7]$. II en ressort que la proportion maximale de carbone $\mathrm{sp}^{3}$ est observée pour une énergie des ions entre 0,1 et $1 \mathrm{keV}$. 
Pour obtenir par ablation par laser $\mathrm{KrF}$, à partir de cible de graphite, des films possédant une forte proportion de liaisons $\mathrm{sp}^{3}$, il est donc nécessaire de travailler à des fluences élevées $\left(\sim 1000 \mathrm{~J} / \mathrm{cm}^{2}\right)$.

Cependant, dans cette situation, on est confronté à l'éjection de particules de graphite, postérieure à l'expansion du panache [8], susceptibles de venir se coller sur la couche en croissance, ce qui constitue un handicap sévère pour certaines applications.

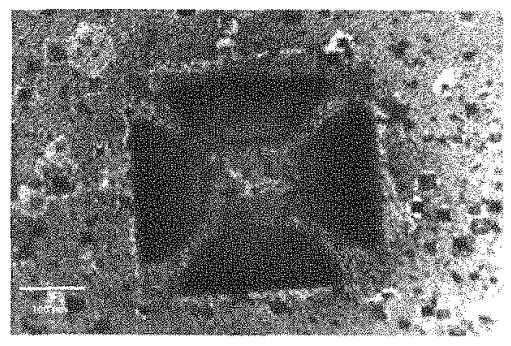

Figure 1: Membrane suspendue de carbone réalisée à forte fluence contaminée par des particules de graphite.

Les phénomènes mis en jeu lors de l'éjection de ces "escarbilles" sont encore mal compris, il semblerait qu'il soit lié au chauffage brutal de la cible par le laser conduisant à une explosion de phase [9]. Plusieurs méthodes sont envisageables pour tenter de résoudre ce problème. Dans la mesure où nous avons constaté expérimentalement une forte corrélation entre l'éjection de ces particules, la densité ainsi que la rugosité de surface de la cible et que notamment l'éjection de particules à partir de carbone vitreux apparaissait pour une fluence seuil nettement supérieure à celle observée pour le graphite, il était intéressant d'étudier en détail l'ablation de cibles de carbone vitreux.

\section{EXPERIENCES}

Le faisceau d'un laser KrF (Lambda Physik EMG $150 \mathrm{MSC}$ ), est focalisé sur une cible de carbone vitreux placée dans une enceinte à ultravide $\left(10^{-7}\right.$ torr). Afin de renouveler la surface de cible irradiée et de conserver des conditions d'ablation constantes au cours du temps, la cible est mise à la fois en rotation et en translation, les impacts laser se répartissent, de façon équidistante, suivant une spirale. La densité de puissance au niveau de la cible est ajustée en déplaçant la lentille de focalisation, compte tenu de la bonne qualité de faisceau du laser utilisé, elle peut atteindre des valeurs de l'ordre de $10^{11} \mathrm{~W} / \mathrm{cm}^{2}$.

Le dispositif d'imagerie employé se compose d'un détecteur ICCD $(578 \times 384$ pixels de $22 \mu \mathrm{m}$ de coté) intensifié Princeton Instrument et de son système de pilotage. Il permet des temps d'intensification de l'ordre de 10ns. Afin de suivre l'évolution d'une espèce donnée de façon sélective, un filtre accordable (VARISPEC) est placé devant l'objectif de la caméra. Le filtre peut être ajusté en longueur d'onde dans le domaine 400 à $720 \mathrm{~nm}$ avec une largeur de bande de $5 \mathrm{~nm}$. L'ensemble est synchronisé avec le laser par l'intermédiaire d'un générateur de délai (Stanford DG535).

Une étude préliminaire par spectroscopie résolue en temps a permis de sélectionner, pour chaque espèce, les raies les plus intenses dans la gamme de travail du filtre permettant ensuite de centrer sa bande passante. Afin d'améliorer le rapport signal sur bruit, chaque image correspond à l'accumulation de cinq prises de vue successives de la camera.

A partir des mesures d'intensité, on remonte au coefficient d'émission volumique par inversion d'Abel, ce qui donne accès, à chaque instant choisi, aux distributions axiales et radiales des espèces émettrices dans le panache d'ablation.

\section{RESULTATS ET DISCUSSION}

Les premières analyses spectroscopiques ont consisté à identifier les espèces radiatives présentes dans le panache pour trois fluences : 15,30 et $70 \mathrm{~J} / \mathrm{cm}^{2}$. Dès la plus basse fluence étudiée, nous n'observons pas les bandes d'émission de $C_{2}^{*}$ (bande de $S w a n$ ). Par contre nous trouvons les raies relatives à $\mathrm{C}^{+*}$ et $\mathrm{C}^{++^{* *}}$.

Une première série d'images a été réalisée pour déterminer la fluence seuil d'apparition des escarbilles dans le cas de l'interaction laser - carbone vitreux. Cette fluence a été trouvée d'environ $80 \mathrm{~J} / \mathrm{cm}^{2}$ pour une 
cible vierge d'impacts à comparer au cas du graphite, pour lequel es escarbilles apparaissent dés $10 \mathrm{~J} / \mathrm{cm}^{2}$, leur nombre et leur vitesse augmentant avec la fluence. [10]

Des chronologies de l'expansion du panache ont été effectuées pour le carbone vitreux en fonction de la fluence pour deux longueurs d'onde $427,465 \mathrm{~nm}$ correspondant respectivement à des raies d'émission de $\mathrm{C}^{+^{*}}$ et $\mathrm{C}^{++^{*}}$. Après symétrisation des images, l'inversion d'Abel permet de déduire la distribution radiale de l'espèce émettrice considérée (Figure 2).

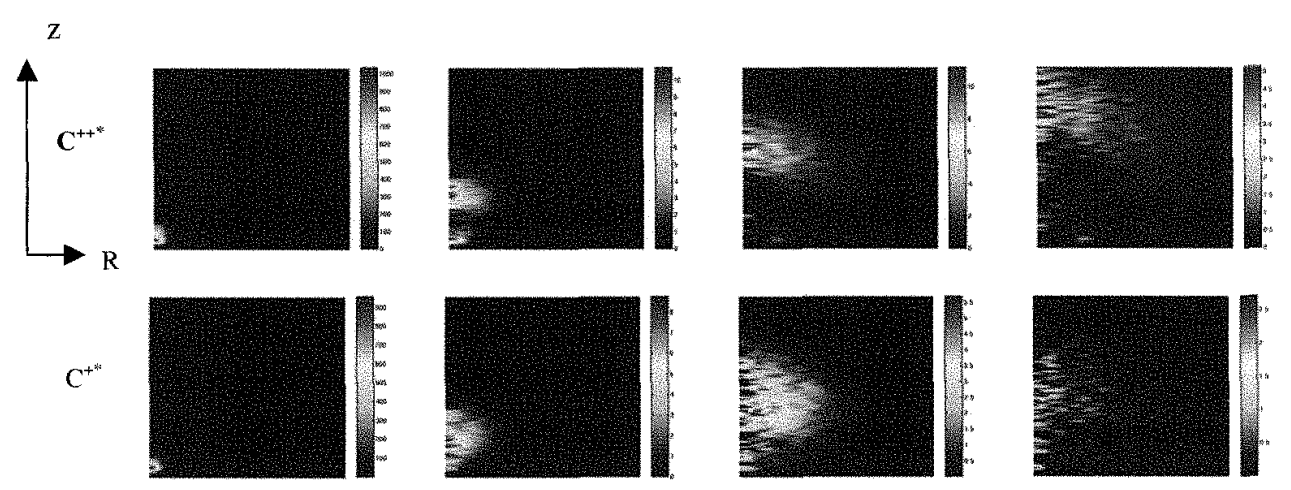

Figure 2: Chronologie(100, 200, 300, 400ns), après inversion d'Abel de la répartition de $\mathrm{C}++^{*}$ et $\mathrm{C}+*$ à $70 \mathrm{~J} / \mathrm{cm}^{2}$. Dimension réelle : hauteur $5 \mathrm{~cm}$, largeur $4 \mathrm{~cm}$.

Dès les premiers instants, l'expansion s'effectue en trois dimensions et comme attendu, la vitesse d'expansion du panache crôt avec la fluence. Pour chaque image, nous avons déterminé la position du barycentre suivant la normale à la cible qui renseigne sur le mouvement d'ensemble des ions et du barycentre parallèlement à la cible qui renseigne sur l'élargissement du panache. Comme le montre la Figure 3 la position du premier évolue linéairement avec le temps. Le même résultat est obtenu pour le deuxième barycentre.

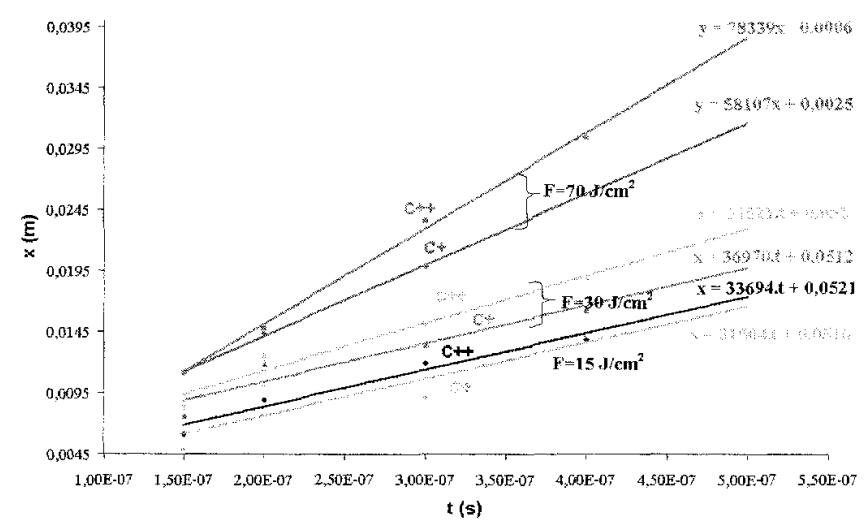

Figure 3 :Evolution de la position du centre de masse des ions $\mathrm{C}^{+*}$ et $\mathrm{C}^{++^{*}}$ pour différentes fluences

On constate sur la Figure 3 que pour toutes les fluences étudiées, les vitesses mesurées des ions $\mathrm{C}^{+t^{*}}$ sont toutes supérieures à celles des ions $\mathrm{C}^{+^{*}}$. Par contre, l'élargissement du panache s'effectue à des vitesses équivalentes pour les ions $\mathrm{C}^{++^{*}}$ et les ions $\mathrm{C}^{+^{*}}$ ce qui implique que la distribution des ions $\mathrm{C}^{++^{*}}$ est plus resserrée sur l'axe d'expansion que celle des ions $\mathrm{C}^{+*}$ et cela d'autant plus que la fluence augmente.

En accord avec les résultats obtenus pour l'ablation du graphite par détecteur d'ions (temps de vol) et par spectroscopie optique [3], on peut distinguer deux populations d'ions :

- la première correspond aux ions les plus rapides créés lors des instants initiaux de l'interaction, accélérés par l'effet de charge d'espace.

- la seconde correspond au "corps" du panache plasma globalement neutre. 
Les résultats présentés dans la Figure 3 correspondent à cette seconde population. Pour obtenir des informations relatives aux ions les plus rapides, il est nécessaire de s'intéresser au déplacement du front du panache. L'évolution de la hauteur du front du panache suit une loi linéaire et comme montré sur la Figure 4 , à la fluence de $70 \mathrm{~J} / \mathrm{cm}^{2}$, les énergies cinétiques des premiers ions $\mathrm{C}^{+^{*}}$ et $\mathrm{C}^{++^{*}}$ sont très voisines et dépassent le $\mathrm{keV}$.

A la fluence de $70 \mathrm{~J} / \mathrm{cm}^{2}$, proche du seuil d'apparition de l'éjection de particules, les ions issus de la cible de carbone vitreux sont nettement plus rapides que ceux éjectés d'une cible de graphite.

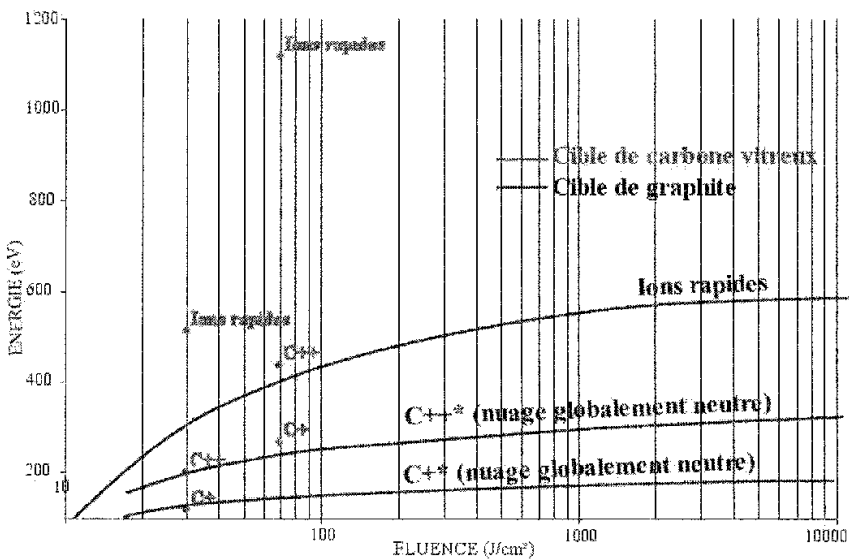

Figure 4: Evolution de l'énergie des ions $\mathrm{C}^{\mathrm{H}^{*}}$ et $\mathrm{C}^{++^{*}}$ en fonction de la fluence pour le graphite et le carbone vitreux

Nous attribuons cet écart important au fait que, dans le cas du graphite, une partie importante de l'énergie apportée par le laser est utilisée pour l'éjection de particules de graphite au détriment de l'énergie cinétique des ions.

Des films ont été déposés sur substrats silicium à partir de cibles de carbone vitreux à $20 \mathrm{~J} / \mathrm{cm}^{2}$ et $70 \mathrm{~J} / \mathrm{cm}^{2}$. Ces films sont de couleur ambre clair et l'examen au microscope optique montre qu'ils sont exempts de particules sur leur surface. Les analyses Raman confirment les qualités adamantines de ces couches, associées à de fortes contraintes compressives [11]. Les spectres obtenus sont très semblables à ceux de films déposés à partir de cible de graphite à des fluences beaucoup plus élevées $\left(\approx 1000 \mathrm{~J} / \mathrm{cm}^{2}\right)$ et présentant un grand nombre de particules en surface.

\section{CONCLUSION}

L'utilisation d'une cible de carbone vitreux apparaît comme une alternative prometteuse à la réalisation de couches DLC non-hydrogénés à fort taux d'hybridation $\mathrm{sp}^{3}$, sans contamination par des escarbilles, pour une fluence de travail n'excèdant pas $100 \mathrm{~J} / \mathrm{cm}^{2}$. Toutefois, il apparaît que ces films sont plus fortement contraints que ceux obtenus à fluence élevée avec une cible graphite.

\section{References}

[1] X. Shi, L. K. Cheah, J. R. Shi, Z. Sun, B. K. Tay, J. Phys,: Cond. Mat. 11 (1999)

[2] J. Bulir, M. Jelinek, J. Non-Cryst. Sol. 188 (1995) 118-124

[3] C. Germain, Thèse, Université de Limoges (1993)

[4] A. A. Voevodin, S. J. P. Laube, Surf. Coat. Tech. 76-77 (1995) 670-674

[5] D. R. McKenzie et al., Diam. Rel. Mat. 1 (1991) 51.

[6] P. J. Fallon, V. S. Veerasamy, C. A. Davis, J. Robertson, Phys. Rev. B 48 (1993) 4877.

[7] Y. Lifshitz, G. D. Lempert, E. Grossman, Diam. Rel. Mat. 4 (1995) 318.

[8] F. Claeyssens, M. N. R. Ashold, E. Sofoulakis, J. Appl. Phys. 91 (2002) 6162

[9] R. Kelly, A. Miotello, , Appl Surf. Sci. 96-98 (1996) 205-215

[10] B. Angleraud, F. Garrelie, F. Tétard, A. Catherinot, Appl Surf. Sci. 138-139 (1999) 507-511

[11] J-K. Snin, C. S. Lee, K-R. Lee, K. Y. Eun, Appl. Phys. Lett. 78 (2001) $631-633$ 